\title{
PROPUESTA DE DISEÑO DE UN RADIO DEFINIDO POR SOFTWARE PARA LA CARACTERIZACIÓN DE LA BANDA IMT
}

\section{DESIGN PROPOSAL OF A SOFTWARE DEFINED RADIO FOR THE IMT BAND CHARACTERIZATION}

\section{MSc. Mónica Espinosa Buitrago, Ing. Víctor Castro Ramírez, Ing. Iván Díaz Pardo. Ing. Mateo González Moreno, Ing. Cesar Villate Barrera, Ing. Gina Roncancio Q.}

Universidad Santo Tomas, Ingeniería de Telecomunicaciones. Grupo de Investigación INVTEL, USTA.

Cra 9. No. 51-11, Bogotá, Cundinamarca, Colombia, Tel.: (+571) 5878797.

E-mail: \{monica.espinosa, victorcastro, ivanediaz, mateo.gonzalez, cesar.villate, gina.roncancio\}@usantotomas.edu.co.

\begin{abstract}
Resumen: En este artículo se define la propuesta de diseño de un radio definido por software para la caracterización o sensado de la banda de telecomunicaciones móviles celulares. Para ello, se definen los sistemas de radiocomunicaciones utilizados en los servicios inalámbricos. Con este fin se caracterizaron las bandas de espectro radioeléctrico denominadas IMT (Internacional Mobile Telecommunication) y se analizan los sistemas de radio definido por software para posteriormente presentar la propuesta de diseño. En donde se definieron las etapas de diseño de la plataforma de gestión, diseño del radio definido por software y el procesamiento de señal que permitirán generar plataformas con sistemas de USPR (Universal Software Radio Peripheral) mediante herramientas como MATLAB y GNU Radio.
\end{abstract}

Palabras clave: Telecomunicaciones, TIC, Espectro Radioeléctrico, SDR, Banda IMT.

\begin{abstract}
This article defines the design proposal a software-defined radio for characterization or sensing of cellular band mobile communications. For this, radio systems are defined for the wireless service. With this, the spectrum bands were characterized like IMT (International Mobile Telecommunication) and will be analyzed the systems of software-defined radio, then will be presented the design proposal. Where defined the design management platform, design software defined radio and signal processing for generate platforms used USPR systems (Universal Software Radio Peripheral) through tools like MATLAB and GNU Radio.
\end{abstract}

Keywords: Telecommunications, ICT, Radioelectric Spectrum, SDR, IMT Band.

\section{INTRODUCCIÓN}

La transmisión de diferentes servicios de telecomunicaciones inalámbricos implica plataformas tecnológicas que soporten los requerimientos que demandan los usuarios. Debido a la diversidad de los servicios, se requieren tecnologías que puedan adaptarse a las necesidades del canal o medio de transmisión o espectro radioeléctrico. La propuesta de diseño de los sistemas de radio definidos por software se basa en la recepción y transmisión de señales y el procesamiento de señal de los dispositivos de transmisión y recepción en la banda de IMT (International Mobile Telecommunications) por sus siglas en inglés, enfatizando en cómo se podría 
desarrollar una caracterización o detección del espectro radioeléctrico en esta banda aplicando radio definido por software.

\section{USO DE ESPECTRO IMT}

Con el objetivo de analizar el uso de espectro IMT es necesario tomar como referencia las bandas establecidas por la UIT (Unión Internacional de Telecomunicaciones).

La Conferencia Mundial de Radiocomunicaciones (CMR) de 1992, 2000 y 2007 [1] ha permitido definir y ser documento de apoyo para el Ministerio de tecnologías de la información y las comunicaciones en la banda IMT. Como se puede observar en la figura 1 [2].

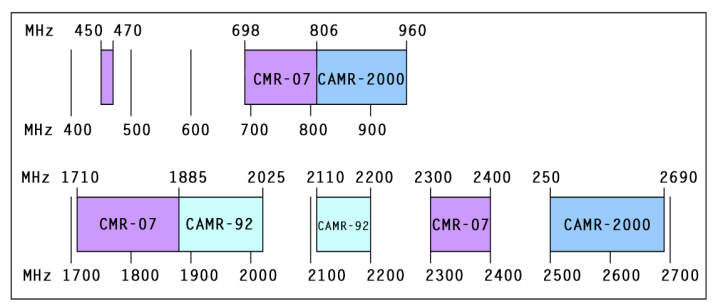

Fig. 1. Bandas Identificadas por la UIT como IMT. Fuente: Ministerio de las TICs a partir de UIT [2].

Las Bandas determinadas desde $850 \mathrm{MHz}$ hasta $2500 \mathrm{MHz}$ están asignadas a los principales operadores de comunicaciones móviles en Colombia:

- Comcel S.A

- Telefónica Móviles S.A

- Movistar

- Colombia Móvil S.A

- ESP

- Tigo

- UNE Telecomunicaciones

- DIRECTV

Estos operadores ofrecen a los usuarios de tecnologías móviles tecnologías de tipo GSM/EDGE, UMTS/HDSPA y 4G/LTE

Al analizar las bandas de IMT se observa una banda denominada dividendo digital [3]. En la evolución de la televisión analógica a digital se generó liberación de una porción de espectro radioeléctrico, a esta liberación y aprovechamiento de espectro se le denomina Dividendo Digital.

En Colombia, ese rango de frecuencias (caracterizada desde 698MHz-806MHz) está siendo ocupado por la Televisión analógica, es por esta razón que el ministerio de Tecnologías de la información y las comunicaciones está adelantando un plan para hacer la migración de los servicios de televisión a dicha Banda [4].

\subsection{Radio definido por software.}

El radio definido por software (SDR) ha sido una revolucionaria tecnología que opera dentro de los sistemas de telecomunicaciones [5], incluyendo aplicaciones en sistemas militares y comerciales. Con un avanzado sistema de procesamiento digital y desarrollo computacional, útil en la transición de sistemas de radio basados en hardware a software, permitiendo la reconfiguración del servicio en operación [6]. Se ha iniciado la explotación científica de estos sistemas, evolucionando la reconfiguración de los servicios críticos, permitiendo la flexibilidad al sistema en operación, adaptando el sistema para los diferentes requerimientos [6]. Los radios se han desarrollo con circuitos integrados (ASICs) que no podrían ser adaptativos por sus arquitecturas cerradas.

Una radio definida por Software (SDR) es un sistema de radiocomunicaciones configurable en diferentes bandas de frecuencia y cuya aplicabilidad en las comunicaciones actuales se ve altamente demandada. Un sistema de SDR está compuesto por diferentes elementos electrónicos que son programados mediante algún software computacional, dichos elementos pueden ser filtros, moduladores, demoduladores, amplificadores, entre otros [7].

La NASA ha adoptado el radio definido por software debido a que este permite la adaptación tecnológica en estándares abiertos [6]. Se observa en la figura 2 [6] una misión desarrollada por la NASA en Space telecommunications radio system (STRS), que ha permitido generar la plataforma de gestión y el uso de radio definido por software [6].

Actualmente las investigaciones en el campo de la radio definida por software están dirigidas a la concepción del sistema de transmisión y la gestión del mismo para permitir la reconfiguración del sistema.

En las redes de área local inalámbricas se observan propuestas de diseño IEEE 802 en [8]. Para sistemas de radio FM se observa la propuesta [8] y las metodologías para definir de tecnologías de radio definido por software [9]. 


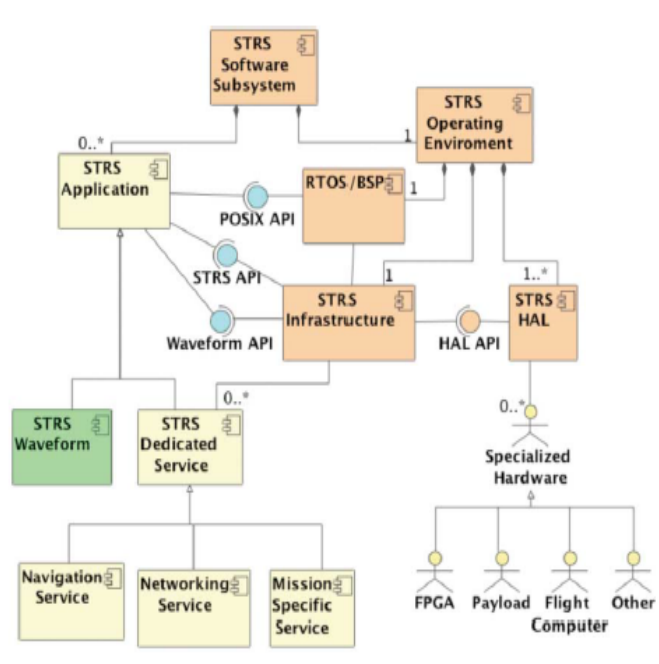

Fig. 2. Misión NASA radio definido por software. Fuente: NASA [6]

\section{PROPUESTA DEL DISEÑO DE UN RADIO DEFINIDO POR SOFTWARE}

Al analizar las bandas IMT se observa que son necesarios sistemas que permitan generar las mediciones para caracterizar la banda con la reacomodación de servicios como en el estudio de la banda UHF [10]. Para ello se han revisado metodologías de detección de espectro radioeléctrico que serán implementadas con radio definido por software [11], [12].

Los sistemas de Universal Software Radio Peripheral (USRP) son una familia de dispositivos fabricados por ETTUS Research [13], según la arquitectura de software, utilizando MATLAB [14] y GNU Radio [15] son dispositivos que serán utilizados para la implementación del diseño y se observan en la Figura 3.

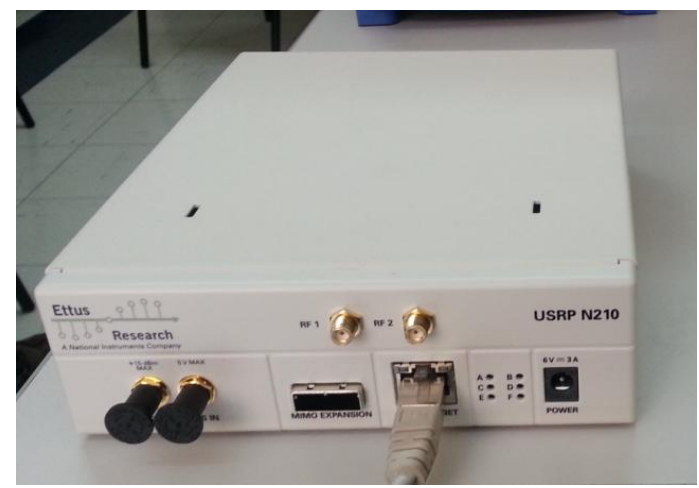

Fig. 3. Equipos diseño de radio definido por software. Fuente: Propia.
En la experimentación e implementación del sistema se utiliza un ordenador con las siguientes características:

- $\quad$ Procesador Xeon 3040 Dual-Core 1.86GHz.

- 2 GB de Memoria RAM

En la Figura 4 se presenta un resumen esquemático de los pasos que comprenden la metodología propuesta para el desarrollo del proyecto. Esta metodología consiste en una base cuantitativa debido a que se pretende aumentar la eficiencia de un sistema determinístico mediante el uso de distintas estrategias y mediciones realizadas a un sistema. En el esquema se pueden observar las distintas fases que comprenden el proyecto y las fuentes a partir de las que serán ejecutadas.

\subsection{Diseño e implementación de la plataforma de gestión.}

La plataforma de gestión está constituida por los algoritmos y programas que permiten el procesamiento de los datos del radio definido por software, esta permite cambios en frecuencias, anchos de banda, espectros en potencia y procesamiento analógico y digital de las señales.

Esta plataforma será implementa por medio de las herramientas de la plataforma GNU Radio. Sus componentes han sido clasificados como interfaces de usuario para el almacenamiento de indicadores de funcionamiento y rutinas de procesamiento encargadas de la manipulación de las señales de información.

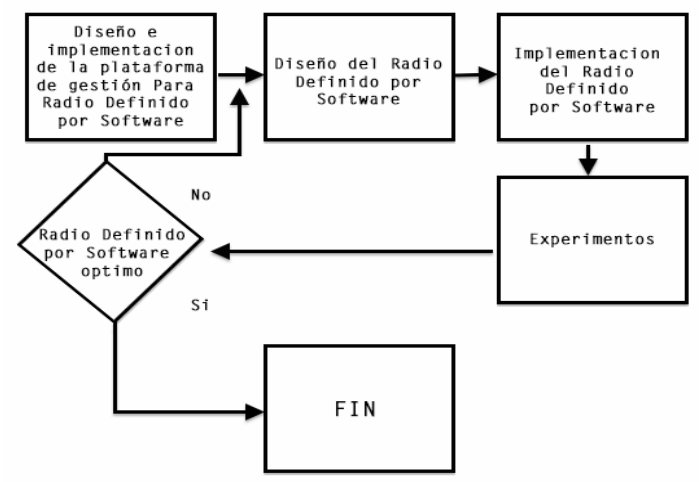

Fig. 4. Metodología del diseño del radio definido por software. Fuente: Propia.

El desarrollo de la propuesta se realiza en GNU radio en donde se observa el primer módulo de configuración en la figura 5 . 
El dispositivo es denominado USPR_Source y permite la comunicación mediante el protocolo Ethernet con dirección IP 192.168.10.2, las muestras son relacionadas con la velocidad de Ethernet y con el ancho de banda requerido, la frecuencia central es modificada por la variable definida en el flow graph de diseño GNU que se observa en la fig. 6.

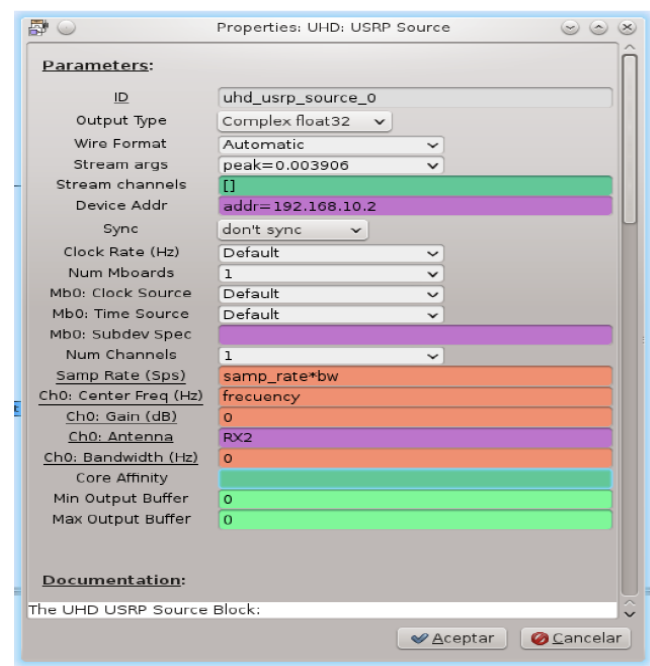

Fig. 5. Configuración bloque USRP Source. Fuente: Propia
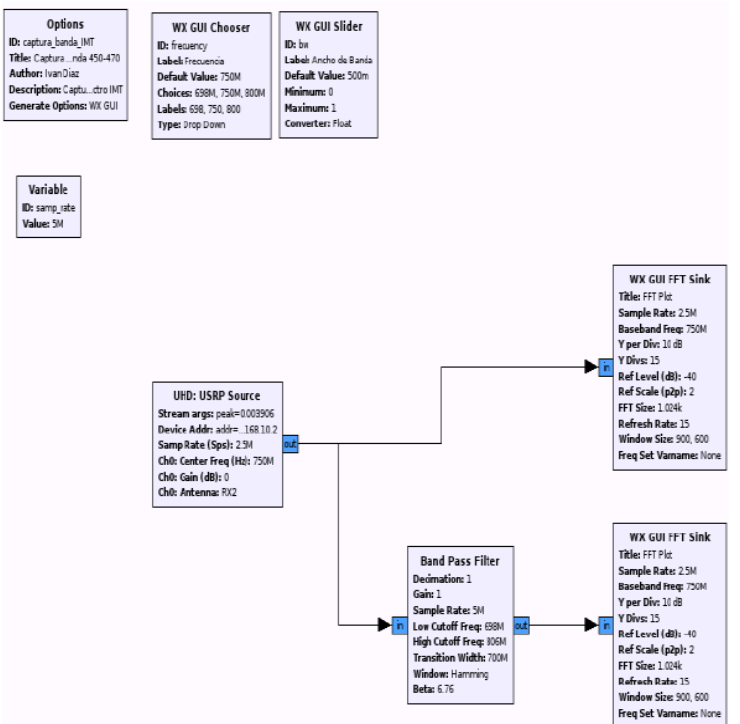

Fig. 6 Bloques GNU Radio. Fuente: Propia

\subsection{Diseño del radio definido por software.}

El diseño del radio definido por software tendrá los siguientes componentes: Sistema de recepción y transmisión de señales, procesamiento de señales.

\subsubsection{Recepción y transmisión de señales.}

Permite recibir las señales del espectro radioeléctrico, determinando las bandas de frecuencia del radio definido por software. Las bandas de frecuencia que han sido definidas en este proyecto son:

\section{- $50 \mathrm{MHz}-2.2 \mathrm{Ghz}$ (Con placa Secundaria WBX).}

- $1.2 \mathrm{GHz}-6 \mathrm{GHz}$ (Con placa Secundaria CBX).

Trabajadas mediante tarjetas hijas USPR para el sistema principal, que permiten cubrir la totalidad de las bandas IMT [2].

\subsubsection{Procesamiento de señal.}

Permite la comunicación con la plataforma de gestión mediante las herramientas MATLAB y GNU Radio y el sistema de transmisión y recepción de señales, desarrollando la comunicación con el procesamiento digital y/o analógico.

En la Figura 8 se observa un análisis desarrollado en GNU que permite filtrar las señales en la primera banda IMT de $698 \mathrm{MHz}$ a $806 \mathrm{MHz}$ CMR07.

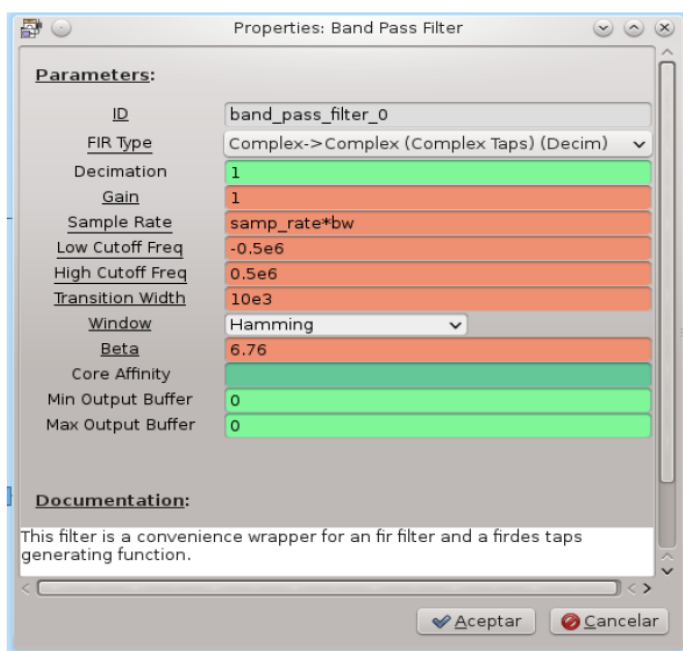

Fig. 7. Desarrollo de código para análisis de Filtros. Fuente: Propia.

El espectro de frecuencia se visualiza fft_sink que se observa en la Figura 8. En la Figura 9 se observa el espectro capturado en banda CMR07, en la parte superior se observa el espectro aplicando el filtrado.

El GNU permite la captura de la señal recibida en archivos con formato *.dat. Estos son procesados 
en MATLAB con el fin de realizar los cálculos de las densidades espectrales de frecuencia.

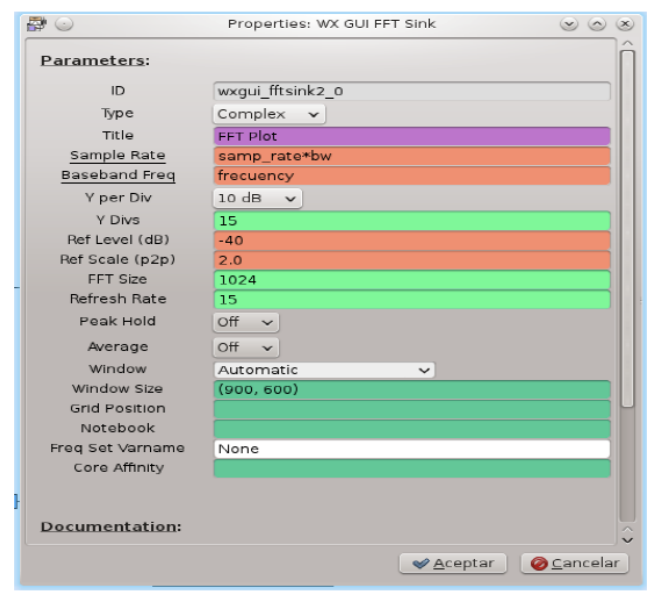

Fig. 8. Desarrollo de Código de la densidad Espectral de Potencia.

Fuente: Propia.

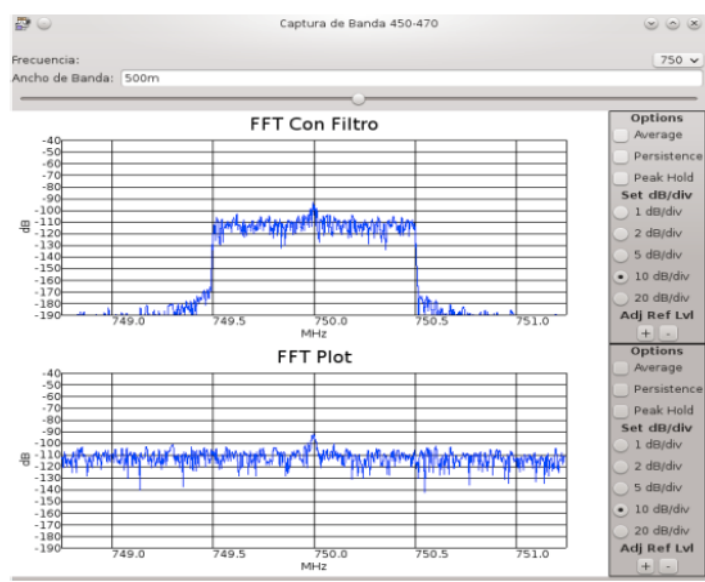

Fig. 9. Flow Graph para captura de Espectro de la primera banda de dividendo digital CMRO7.

Fuente: Propia

\subsection{Implementación del radio definido por software.}

Se establece la comunicación del radio definido por software entre el equipo de transmisión y recepción. Logrando sincronización, codificación, decodificación y procesamiento de la señal tanto en transmisión como en recepción.

En esta fase se implementarán los modelos para la detección espectral en radio definido por software según [11] [10].

Los archivos de recepción se procesaran en MATLAB como se observa en la figura 10 con el fin de aplicar los modelos de detección espectral.

$$
\begin{gathered}
\text { Densidad } 1=\mathrm{abs}\left(\text { Fourier) } .^{\wedge} 2 ;\right. \\
\text { Densidadfl }=\mathrm{sum}(\text { Densidad } 1) ; \\
\text { frec_vec(cont)=f; } \\
\text { den_vec(cont)= Densidadf1; } \\
\text { Fig. } 10 \text { Densidad Espectral. } \\
\text { Fuente: } \text { Propia }
\end{gathered}
$$

\subsection{Experimentos.}

Modificando el bloque de procesamiento de señal según las variables del ancho de banda y espectro de potencia de transmisión, se establecen diferentes sistemas que permitirán evaluar las bandas IMT.

\subsection{Comparaciones.}

Una vez superada las fases de experimentación y se obtengan algunos radio definidos por software candidatos, se proceden a realizar comparaciones entre las implementaciones de los resultados a nivel de las bandas IMT.

\section{CONCLUSIONES}

Se desarrolló una revisión de las bandas IMT analizando la asignación según la UIT en la eficiencia espectral realizando una propuesta metodológica para definir radios definidos por software para esta banda, mediante el uso equipos de marca Ettus que cubren los requerimientos de la banda IMT.

Se definieron las etapas a nivel del radio definido por software tales como el diseño plataforma de gestión, diseño del radio definido por software y procesamiento de señal, que permitirán generar plataformas flexibles haciendo uso de estas tecnologías.

Como trabajo futuro se desarrollarán las plataformas de radio definido por software con sistemas USPR que permitan cubrir las diferentes necesidades sobre IMT, tales como caracterización o detección de la banda.

\section{AGRADECIMIENTOS}

Universidad Santo Tomás, Facultad de Ingeniería de Telecomunicaciones, Bogotá y en particular al Grupo de investigación INVTEL, USTA 


\section{REFERENCIAS}

[1]. F. Leite, R. Engelman, S. Kodama, H. Mennenga, and S. Towaij, "Regulatory considerations relating to IMT-2000," Pers. Commun. IEEE, vol. 4, no. 4, pp. 14-19, Aug. 1997.

[2]. Ministerio de las TICs. "Documento de politica banda 2.500 a $2.690 \mathrm{MHz}$." Mar2010.

[3]. M. T. García Leiva, "El dividendo digital: desafíos, oportunidades y posiciones nacionales," 2009.

[4]. J. Benavides, F. Castro, and L. Tamayo, "Promoción de la competencia en la telefonía móvil de Colombia," Fedesarrollo Regul., 2012.

[5]. J. M. Costa, "Las tecnologías de radiocomunicaciones de la próxima generación flexibilizarán la utilización del espectro," Actual. UIT, no. 2, pp. 23-25, 2008.

[6]. R. C. Reinhart, S. K. Johnson, T. J. Kacpura, C. S. Hall, C. R. Smith, and J. Liebetreu, "Open Architecture Standard for NASA's Software-Defined Space Telecommunications Radio Systems," Proc. IEEE, vol. 95, no. 10, pp. 1986-1993, 2007.

[7]. A. A. Tabassam, F. A. Ali, S. Kalsait, and M. U. Suleman, "Building Software-Defined Radios in MATLAB Simulink - A Step Towards Cognitive Radios," in Computer Modelling and Simulation (UKSim), 2011 UkSim 13th International Conference on, 2011, pp. 492-497.

[8]. H. J. Eslava Blanco, L. A. Rojas Castellar, and M. L. Tello Castaneda, "Modelo de un sistema de radio digital utilizando infraestructura de FM comercial y recomendaciones de implementación en Colombia," Rev. Tecnura, vol. 11, No. 22, pp. 16-27, 2008.
[9]. W. H. Tuttlebee, Software defined radio: enabling technologies. John Wiley \& Sons, 2003.

[10]. H. Paz Penagos, M. Bohórquez, D. Rodríguez, D. Maldonado, C. Sánchez, and C. Galarza, "Tecnología radio cognitiva en la banda ultra high frequency (UHF)," Rev. Tecnura, vol. 18, no. 39, pp. 138-151, 2013.

[11]. K. Seshukumar, R. Saravanan, and M. Suraj, "Spectrum sensing review in cognitive radio," in Emerging Trends in VLSI, Embedded System, Nano Electronics and Telecommunication System (ICEVENT), 2013 International Conference on, 2013, pp. 1-4.

[12]. T. Yucek and H. Arslan, "A survey of spectrum sensing algorithms for cognitive radio applications," Commun. Surv. Tutor. IEEE, vol. 11, no. 1, pp. 116-130, 2009.

[13]. J. de J. R. Uribe, T. M. Bojac., and Cè. H. C. S.nchez, "CaracterizaciÛn de la plataforma de radio definido por software USRP N210WBX," Rev. GTI, vol. 12, no. 34, 2014.

[14]. A. A. Tabassam, F. A. Ali, S. Kalsait, and M. U. Suleman, "Building Software-Defined Radios in MATLAB Simulink-A Step Towards Cognitive Radios," in Computer Modelling and Simulation (UKSim), 2011 UKSim 13th International Conference on, 2011, pp. 492-497.

[15]. M. A. Sarijari, A. Marwanto, N. Fisal, S. K. S. Yusof, R. A. Rashid, and M. H. Satria, "Energy detection sensing based on GNU radio and USRP: An analysis study," in Communications (MICC), 2009 IEEE 9th Malaysia International Conference on, 2009, pp. 338-342. 